\title{
Casimir Effect in Fluids above the Isotropic-Lamellar Transition
}

\author{
Nariya Uchida \\ Department of Physics, Kyoto University, Kyoto 606, Japan
}

(Submitted 2 Sep 2000; revised 14 Dec 2000; published 31 Oct 2001)

\begin{abstract}
We study fluctuation-induced interaction in confined fluids above the isotropic-lamellar transition. At an ideally continuous transition, the disjoining pressure has the asymptotic form $\Pi(d \rightarrow \infty) \approx$ $-C k_{B} T q_{0}^{2} / d$, where $d$ is the interwall distance, $q_{0}$ is the wavenumber of the scattering peak, and $C=1 /(4 \pi)$ in the strong anchoring limit. The long-rangedness is enhanced due to continuous distribution of soft modes in the q-space. An unconventionally strong Casimir force with a range of several lamella thicknesses is realistic above the transition. We also find an oscillatory force profile near a surface-induced transition.
\end{abstract}

PACS numbers: 68.15.+e, 64.60.Cn, 61.25.Hq, 82.70.-y

Fluids in confined geometries show a variety of phenomena that are not observed in bulk. Among them, thermal-fluctuation-induced interactions between boundary walls or objects immersed in the fluid have attracted much attention. They are called the Casimir forces by analogy with the quantum original [1]. The forces are long-ranged in systems with soft modes, such as critical simple fluids [2.,3] and liquid crystals [4., [], with the decay law and amplitude modulated by various surface effects [3,6, 67. Recent evidence [8,9, 10] supports the view that the interaction is ubiquitous in correlated fluids 11].

In this Letter, we address the effect in structured fluids above the isotropic-lamellar (I-L) transition. The model system we consider has two characteristic lengths, which describe decay and oscillation of the correlation function. Physical realizations of the model include block copolymers in the disordered phase and bicontinuous microemulsions. Films of block copolymers have constituted the subject of numerous papers 112, 13, 14, 15, 16. Most of them focus on the lamellar phase, while a few theoretical works treat the system above the I-L transition 15, 16. The latter works discuss the effect of a surface field, which induces a mean-field interaction between boundary walls. In comparison to the case of copolymers, much less is known about confined fluids containing short-chain surfactants [17, 18, 19]. A meanfield study with a standard Ginzburg-Landau model of microemulsions found a surface-induced I-L transition that preempts the bulk transition [17].

The bulk property of the system we assume is described by the Hamiltonian

$$
\mathcal{H}_{b}=\frac{\epsilon}{2} \int \frac{d \mathbf{q}}{(2 \pi)^{3}}\left[\left(q^{2}-q_{0}^{2}\right)^{2}+p_{0}^{4}\right]\left|\phi_{\mathbf{q}}\right|^{2},
$$

where $\epsilon$ is a constant, $\phi$ is the order parameter, and $p_{0}$ and $q_{0}$ are characteristic wavenumbers that generally depend on the temperature. This and similar types of Hamiltonians, with or without additional nonlinear terms, have been applied to various kinds of complex fluids 20,21, 22, 23]. For symmetric AB-block copolymer melts, for instance, $\phi$ represents the excess of A- monomer's (say) volume fraction with respect to its spatial average, and the parameters are given by $\epsilon=$ $0.0327 k_{B} T R_{g} / N^{1 / 2}, q_{0}=1.95 / R_{g}$, and $p_{0}=1.43\left[\left(\chi_{c}-\right.\right.$ $\chi{ } N]^{1 / 4} / R_{g}$. Here, $R_{g}$ is the chain's gyration radius, $N$ is the polymerization index, $\chi$ is the Flory interaction parameter, and $\chi_{c}=10.5 / N$ locates the mean-field I-L transition 20]. To be general, we shall use the ratio $p_{0} / q_{0}$ as the measure of distance from the transition temperature.

We consider a confined film of thickness $d$ and of macroscopic area. The total Hamiltonian is the sum of bulk and surface contributions, $\mathcal{H}=\mathcal{H}_{b}+\mathcal{H}_{s}$. We write the bulk Hamiltonian (11) in the real space as

$$
\mathcal{H}_{b}=\frac{\epsilon}{2} \int_{0<z<d} d \mathbf{r}\left[\left(\nabla^{2} \phi\right)^{2}-2 q_{0}^{2}(\nabla \phi)^{2}+\left(p_{0}^{4}+q_{0}^{4}\right) \phi^{2}\right] .
$$

In this form, the Hamiltonian is equivalent to the Teubner-Strey model of microemulsions [21] in the temperature region between the bicontinuous-lamellar transition and the Lifshitz point. For chemically neutral and identical walls, the surface part of the Hamiltonian can be expanded up to the second order in $\phi$ and $\nabla \phi$ as 24]

$$
\mathcal{H}_{s}=\int d \mathbf{r}\left[\omega_{s} \phi^{2}+g_{s}(\nabla \phi)^{2}\right][\delta(z)+\delta(z-d)] .
$$

The free energy of an elastic medium in a finite geometry can be computed by several different techniques for regularizing a divergent sum [5,6,6,25]. The analysis reduces to a 1D problem for the in-plane Fourier modes $\phi_{\mathbf{q}_{\perp}}(z)=\int d \mathbf{r}_{\perp} \exp \left(-i \mathbf{q}_{\perp} \mathbf{r}_{\perp}\right) \phi(\mathbf{r})$, where $\mathbf{r}_{\perp}=(x, y)$ and $\mathbf{q}_{\perp}=\left(q_{x}, q_{y}\right)$. The partition function for each inplane mode is given by

$$
Z_{\mathbf{q}_{\perp}}=\int d \mathbf{u} \int d \mathbf{v} \int_{\mathbf{u}, \mathbf{v}} \mathcal{D} \phi_{\mathbf{q}_{\perp}} e^{-\left[\mathcal{H}_{b, \mathbf{q}_{\perp}}+\left(\omega_{s}+g_{s} q_{\perp}^{2}\right) \mathbf{u}^{2}+g_{s} \mathbf{v}^{2}\right] / k_{B} T} .
$$

Here, $\mathcal{H}_{b, \mathbf{q}_{\perp}}$ is the Fourier component of $\mathcal{H}_{b}$ and $\int_{\mathbf{u}, \mathbf{v}} \mathcal{D} \phi_{\mathbf{q}_{\perp}}$ means that the functional integral should be taken over the paths that satisfy

$$
\left(\phi_{\mathbf{q}_{\perp}}(0), \phi_{\mathbf{q}_{\perp}}(d)\right)=\mathbf{u}
$$


and

$$
\left(\phi_{\mathbf{q}_{\perp}}^{\prime}(0), \phi_{\mathbf{q}_{\perp}}^{\prime}(d)\right)=\mathbf{v}
$$

where $^{\prime}=\partial / \partial z$. The path integral for a quadratic Hamiltonian that contains a squared second-derivative has been calculated by Kleinert [26. Following his work, we decompose each fluctuation path that satisfies the conditions (5) and (6) into two parts as $\phi_{\mathbf{q}_{\perp}}=\phi_{m, \mathbf{q}_{\perp}}+\delta \phi_{\mathbf{q}_{\perp}}$, where $\phi_{m, \mathbf{q}_{\perp}}=\phi_{m, \mathbf{q}_{\perp}}(z ; \mathbf{u}, \mathbf{v})$ is defined as the path that minimizes $\mathcal{\mathcal { H }}_{b, \mathbf{q}_{\perp}}$ under the same boundary conditions. Then the Hamiltonian is decomposed as

$$
\begin{array}{r}
\mathcal{H}_{b, \mathbf{q}_{\perp}}=\mathcal{I}_{\mathbf{q}_{\perp}}+\mathcal{J}_{\mathbf{q}_{\perp}}, \\
\mathcal{I}_{\mathbf{q}_{\perp}}=\frac{\epsilon}{2} \int_{0}^{d} d z\left\{\left|\delta \phi_{\mathbf{q}_{\perp}}^{\prime \prime}\right|^{2}+2\left(q_{\perp}^{2}-q_{0}^{2}\right)\left|\delta \phi_{\mathbf{q}_{\perp}}^{\prime}\right|^{2}\right. \\
\left.+\left[\left(q_{\perp}^{2}-q_{0}^{2}\right)^{2}+p_{0}^{4}\right]\left|\delta \phi_{\mathbf{q}_{\perp}}\right|^{2}\right\}, \\
\mathcal{J}_{\mathbf{q}_{\perp}}=\frac{\epsilon}{2}\left[\phi_{m, \mathbf{q}_{\perp}}^{\prime} \phi_{m,-\mathbf{q}_{\perp}}^{\prime \prime}-\phi_{m, \mathbf{q}_{\perp}} \phi_{m,-\mathbf{q}_{\perp}}^{\prime \prime \prime}\right. \\
\left.+2\left(q_{\perp}^{2}-q_{0}^{2}\right) \phi_{m, \mathbf{q}_{\perp}} \phi_{m,-\mathbf{q}_{\perp}}^{\prime}\right]_{0}^{d} .
\end{array}
$$

The latter part can be expressed in terms of the boundary values as $\mathcal{J}_{\mathbf{q}_{\perp}}=\mathbf{a} \cdot \mathbf{M} \cdot \mathbf{a}$, where $\mathbf{a}=\left(u_{1}, u_{2}, v_{1}, v_{2}\right)$ and $\mathrm{M}=\mathrm{M}\left(q_{\perp}, d\right)$ is a $4 \times 4$ matrix whose calculation is straightforward. Accordingly, the partition function is factorized into "bulk" and "surface" parts as

$$
\begin{aligned}
Z_{\mathbf{q}_{\perp}} & =Z_{b, \mathbf{q}_{\perp}} \cdot Z_{s, \mathbf{q}_{\perp}}, \\
Z_{b, \mathbf{q}_{\perp}} & =\int_{\mathbf{0}, \mathbf{0}} \mathcal{D} \delta \phi_{\mathbf{q}_{\perp}} e^{-\mathcal{I}_{\mathbf{q}_{\perp}} / k_{B} T}, \\
Z_{s, \mathbf{q}_{\perp}} & =\left(\operatorname{det} \frac{\mathbf{M}+\left(\omega_{s}+g_{s} q_{\perp}^{2}\right) \mathrm{E}_{\mathbf{u}}+g_{s} \mathrm{E}_{\mathbf{V}}}{\pi k_{B} T}\right)^{-1 / 2}, \\
\mathbf{E}_{\mathbf{u}} & =\operatorname{diag}(1,1,0,0), \\
\mathbf{E}_{\mathbf{V}} & =\operatorname{diag}(0,0,1,1) .
\end{aligned}
$$

The bulk factor can be computed using Lagrange multipliers that impose the boundary conditions $\delta \phi_{\mathbf{q}_{\perp}}=$ $\delta \phi_{\mathbf{q}_{\perp}}^{\prime}=0$ [26]. In order to extract the interaction part of the free energy, we should regularize the partition function as $\widehat{Z}_{\mathbf{q}_{\perp}}(d)=\lim _{D \rightarrow \infty}\left[Z_{\mathbf{q}_{\perp}}(d) Z_{\mathbf{q}_{\perp}}(D-\right.$ d) $\left./ Z_{\mathbf{q}_{\perp}}(D / 2)^{2}\right]$, so that $\widehat{Z}_{\mathbf{q}_{\perp}}(\infty)=1$ [25]. Applying this to $Z_{b, \mathbf{q}_{\perp}}$, we have

$$
\begin{aligned}
\widehat{Z}_{b, \mathbf{q}_{\perp}}(d) & =\frac{e^{k_{+} d}}{2}\left[\sinh ^{2}\left(k_{+} d\right)-\frac{k_{+}^{2}}{k_{-}^{2}} \sin ^{2}\left(k_{-} d\right)\right]^{-1 / 2}, \\
k_{ \pm} & =k_{ \pm}\left(q_{\perp}\right) \\
& =\sqrt{\frac{1}{2}\left[\sqrt{\left(q_{\perp}^{2}-q_{0}^{2}\right)^{2}+p_{0}^{4}} \pm\left(q_{\perp}^{2}-q_{0}^{2}\right)\right]}
\end{aligned}
$$

In the same way, we obtain the regularized surface partition function $\widehat{Z}_{s, \mathbf{q}_{\perp}}$, which has a voluminous expression that cannot be written down here. The interaction free energy per area is obtained as

$$
F=-k_{B} T \int \frac{d \mathbf{q}_{\perp}}{(2 \pi)^{2}}\left(\ln \widehat{Z}_{b, \mathbf{q}_{\perp}}+\ln \widehat{Z}_{s, \mathbf{q}_{\perp}}\right) .
$$

First we focus on the bulk contribution by taking the "strong anchoring" limit $\omega_{s} \rightarrow \infty, g_{s} \rightarrow \infty$, for which $\widehat{Z}_{s, \mathbf{q}_{\perp}}=1$. We show the result in terms of the disjoining pressure $\Pi=-\partial F / \partial d$. At the critical point $p_{0}=0$, the contributions to the pressure from the regions $q_{\perp}<q_{0}$ and $q_{\perp}>q_{0}$ are given by, respectively,

$$
\begin{gathered}
\Pi_{<}=-\frac{k_{B} T}{2 \pi d^{3}} \int_{0}^{q_{0} d} d s \frac{s^{2}(s-\sin s \cos s)}{s^{2}-\sin ^{2} s} \\
\left\{\begin{array}{lc}
\approx-\frac{k_{B} T q_{0}^{2}}{2 \pi d}, & q_{0} d \ll 1, \\
\approx-\frac{k_{B} T q_{0}^{2}}{4 \pi d}, & q_{0} d \gg 1,
\end{array}\right.
\end{gathered}
$$

where $s$ is for $\sqrt{q_{0}^{2}-q_{\perp}^{2}} d$, and

$$
\begin{aligned}
\Pi_{>} & =-\frac{k_{B} T}{2 \pi d^{3}} \int_{0}^{\infty} d t \frac{t^{2}\left(e^{-t} \sinh t+t^{2}-t\right)}{\sinh ^{2} t-t^{2}} \\
& =-0.5463 \ldots \times \frac{k_{B} T}{d^{3}},
\end{aligned}
$$

where $t$ is for $\sqrt{q_{\perp}^{2}-q_{0}^{2}} d$. Note that the total force $\Pi_{<}+\Pi_{>}$is proportional to $1 / d$ at large distances. To my knowledge, it is more long-ranged than any (thermal or quantum) Casimir interaction ever predicted for a 3D system. As seen from Eq.(18), the origin of the 1/d tail lies in that every in-plane mode with a wavenumber $q_{\perp}<q_{0}$ has a long-range correlation. It is further reduced to the fact that the bulk soft modes of the system are distributed over the surface of the sphere $|\mathbf{q}|=q_{0}$. This is in contrast to the case of a critical simple fluid, in which the soft mode is located on the single point $\mathbf{q}=\mathbf{0}$ and the Casimir force is proportional to $d^{-3}$ (in the harmonic approximation). The extra factor $d^{2}$ in the present system is thus explained by the dimensionality of distribution of soft modes in the q-space. Shown in Fig.1(a) is the force profile at the critical point.

In real systems, nonlinearity in the free energy makes the I-L transition weakly first order [27], and hence we do not expect to see the truly long-ranged force. For block copolymers, for instance, we estimate the typical and effective value of $p_{0} / q_{0}$ at the transition to be 0.4 . Shown in Fig.1(b) is the profile of the scaled pressure $\Pi d^{3} / k_{B} T$ at finite values of $p_{0} / q_{0}$. Even considerably above the transition, the force has a range of several times $\pi / q_{0}$, the lamella thickness. A weak oscillation arises from the region $q_{\perp}<q_{0}$, while the contribution from the region $q_{\perp}>q_{0}$ is always monotonic. Note also the large magnitude of the interaction; the scaled pressure in its decay range is typically 10 times larger than the value $\Pi d^{3} / k_{B} T=-0.096$ for nematic liquid crystals in the one-constant approximation [5]. 
Next we study the role of fluctuations at boundaries. For the present model with a vanishing surface potential $\left(\omega_{s}=g_{s}=0\right)$, it is known that the mean-field solution $\phi=0$ is destabilized above the bulk I-L transition [17]. The instability remains for a small but finite surface potential. I calculated the region of surface parameters for which the homogeneous mean-field solution is stable at any temperature above the bulk I-L transition. It is plotted in Fig.2(a) in terms of the dimensionless parameters $\Omega_{s}=\omega_{s} /\left(\epsilon q_{0}^{3}\right)$ and $G_{s}=g_{s} /\left(\epsilon q_{0}\right)$. Shown in Fig.2(b) is the disjoining pressure for different values of $\left(\Omega_{s}, G_{s}\right)$ in the stable region and at the bulk transition temperature. The force has the asymptotic behavior $\lim _{d \rightarrow \infty} \Pi d /\left(k_{B} T q_{0}^{2}\right)=-C$, where $C$ is a positive number that weakly depends on $\left(\Omega_{s}, G_{s}\right)$. An oscillation in $\Pi(d)$ appears for a small surface potential, and is amplified as we approach the instability line. On the line, the quantity $\Pi d /\left(k_{B} T q_{0}^{2}\right)$ is almost periodic in $d$ except at small distances. The peak positions are approximately given by $d=(n \pi+\psi) / q_{0}$, where $n$ is any integer and the phase shift $\psi$ moves over the region $[0, \pi]$ as we move over the instability line. To see the origin of the oscillation, we consider the simplifying limit $\left(\Omega_{s}, G_{s}\right)=(\infty, 0)$, which is an endpoint of the instability line. The interaction free energy in this limit reads

$$
\begin{gathered}
F=\frac{k_{B} T}{2 \pi} \int_{0}^{\infty} q_{\perp} d q_{\perp}\left[\ln \left|2 \sin \sqrt{q_{0}^{2}-q_{\perp}^{2}} d\right| \theta\left(q_{0}-q_{\perp}\right)\right. \\
\left.+\ln \left(1-e^{-2 \sqrt{q_{\perp}^{2}-q_{0}^{2}} d}\right) \theta\left(q_{\perp}-q_{0}\right)\right] .
\end{gathered}
$$

Although it is a continuous function of $d$, its gradient (and so $\Pi(d))$ logarithmically diverges at $d=n \pi / q_{0}(>$ $0)$, due to the contribution of the mode with $\left(q_{\perp}, q_{z}\right)=$ $\left(0, q_{0}\right)$. This is closely related to the fact that the mode $\phi \propto \sin \left(q_{0} z\right)$, which is soft in infinite bulk, is allowed by the boundary condition $\phi(0)=\phi(d)=0$ only when $d=n \pi / q_{0}$. When we gradually increase $d$ to cross one of the node points, the soft mode is abruptly allowed and then prohibited again, which corresponds to the rise and drop of the disjoining pressure. For physical (finite) values of $\Omega_{s}$, boundary constraints are not strict and the oscillation in $\Pi(d)$ is not singular 28 .

Up to now, we have assumed that the walls are ideally neutral. In general, the walls have a specific interaction with the fluid, which is most simply described by the linear Hamiltonian $\Delta \mathcal{H}_{s}=\int d \mathbf{r} h_{s} \phi[\delta(z)+\delta(z-d)]$. The surface field $h_{s}$ induces a mean-field interaction between the walls. Let us compare its magnitude with that of the Casimir interaction. To be specific, we consider symmetric diblock copolymer melts with $N=10^{3}, a=0.5$ $\mathrm{nm}$, and $T=400 \mathrm{~K}$, for which the lamella thickness $\pi / q_{0}$ equals $11 \mathrm{~nm}$. For $\Omega_{s}=G_{s}=h_{s} /\left(\epsilon q_{0}^{3}\right)=1.0$, $p_{0} / q_{0}=0.4$, and $d=4.7 \pi / q_{0}=50 \mathrm{~nm}$, minimization of $\mathcal{H}=\mathcal{H}_{b}+\mathcal{H}_{s}+\Delta \mathcal{H}_{s}$ gives the surface value of the order parameter $\phi=0.54$ and the mean-field pressure $\Pi_{\mathrm{mf}}=-11 \mathrm{~Pa}$ 29]. On the other hand, the Casimir pressure for the same parameters and at the same dis- tance is $\Pi_{\mathrm{ca}}=-69 \mathrm{~Pa}$. The ratio $\Pi_{\mathrm{ca}} / \Pi_{\mathrm{mf}}=6.3$ shows that fluctuation effect on the total structural force is significant within several lamella thicknesses. It should be mentioned that harmonic approximation to the free energy is valid only for a weak surface field. It would be realized, for instance, by use of substrates coated by a thin sheet of random copolymers 14]. For a strong surface field, the order parameter is saturated at the walls where there appear wetting layers of the lamellar phase [15]. In that case, the above estimate should be applicable to the force between the surfaces of the wetting layers.

To summarize, we predict an unconventionally strong thermal Casimir effect in structured fluids above the isotropic-lamellar transition. At the ideally continuous transition, the force has a long-range tail proportional to $1 / d$, which is due to the two-dimensional distribution of bulk soft modes in the q-space. The qualitative result should commonly hold for model systems in which the bulk structure factor diverges at a finite wavenumber [30]. Although the force cannot be genuinely longranged in a real system, one should be able to access the temperature region where the force has a range of several layer thicknesses, within which it is much stronger than the conventional Casimir force at criticality. This implies that it also dominates other dispersion forces, such as the van der Waals interaction, which is often comparable to the critical Casimir force [7,8,9]. We have also studied the role of surface potential. Within a standard model of microemulsions, the force profile shows an oscillation near the surface-induced I-L transition. The oscillation originates in resonance of a soft mode in the slab geometry. A measurement of structural force near the spongelamellar transition has reported an oscillatory force superimposed on an attractive background 31. However, more systematic study with controlled surface properties seems necessary to extract the fluctuation part of the interaction. I wish the present work to stimulate further experimental investigation.

I thank K. Sekimoto and K. Sato for useful discussions. This work is supported in part by the Grant-in-Aid for Scientific Research from Japan Society for the Promotion of Science.

[1] H. B. G. Casimir, Proc. K. Ned. Akad. Wet. 51, 793 (1948).

[2] M. E. Fisher and P. G. de Gennes, C. R. Acad. Sci. Ser. B 287, 207 (1978).

[3] M. Krech, The Casimir Effect in Critical Systems (World Scientific, Singapore, 1994) and references therein.

[4] L. V. Mikheev, Zh. Éksp. Teor. Fiz. 96, 632 (1989) [Sov. Phys. JETP 69, 358 (1989)].

[5] A. Ajdari et al., Phys. Rev. Lett. 66, 1481 (1991); A. Ajdari et al., J. Phys. II 2, 487 (1992).

[6] H. Li and M. Kardar, Phys. Rev. Lett. 67, 3275 (1991); Phys. Rev. A 46, 6490 (1992).

[7] P. Ziherl et al., Phys. Rev. Lett. 82, 1189 (1999); ibid 84, 1228 (2000). 
[8] A. Mukhopadhyay and B. M. Law, Phys. Rev. Lett. 83, 772 (1999).

[9] R. Garcia and M. H. W. Chan, Phys. Rev. Lett. 83, 1187 (1999).

[10] F. Vandenbrouck et al., Phys. Rev. Lett. 82, 2693 (1999); see also Ref.[7] (latter).

[11] M. Kardar and R. Golestanian, Rev. Mod. Phys. 71, 1233 (1999).

[12] M. S. Turner, Phys. Rev. Lett. 69, 1788 (1992).

[13] M. Sikka et al., Phys. Rev. Lett. 70, 307 (1993).

[14] G. J. Kellogg et al., Phys. Rev. Lett. 76, 2503 (1996).

[15] S. T. Milner and D. C. Morse, Phys. Rev. E 54, 3793 (1996).

[16] K. Binder et al., J. Phys. II 7, 1353 (1997).

[17] F. Schmid and M. Schick, Phys. Rev. E 48, 1882 (1993).

[18] R. Hołyst and P. Oswald, Phys. Rev. Lett. 79, 1499 (1997).

[19] A. Ciach et al., Europhys. Lett. 45, 495 (1999).

[20] L. Leibler, Macromolecules 13, 1602 (1980).

[21] M. Teubner and R. Strey, J. Chem. Phys. 87, 3195 (1987).

(a)

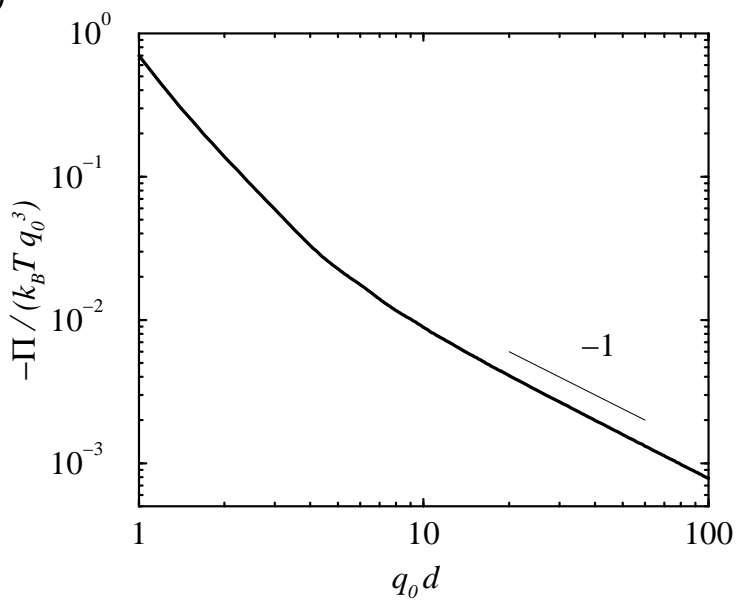

(b)

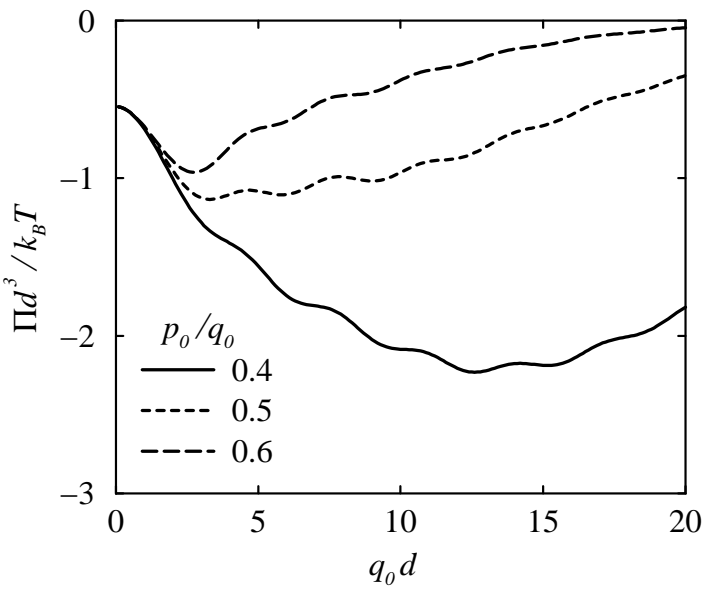

FIG. 1. Profile of the Casimir force in the strong anchoring limit $\omega_{s} \rightarrow \infty, g_{s} \rightarrow \infty$. (a) Log-log plot of the absolute value $|\Pi|$ at the critical point $p_{0}=0$. (b) Scaled disjoining pressure above the transition temperature.
[22] D. Andelman et al., J. Chem. Phys. 86, 3673 (1987).

[23] J. Swift, Phys. Rev. A 14, 2274 (1976).

[24] G. Gompper and S. Zschocke, Phys. Rev. A 46, 4836 (1992).

[25] M. Fierz, Helv. Phys. Acta 33, 855 (1960).

[26] H. Kleinert, J. Math. Phys. 27, 3003 (1986)

[27] S. A. Brazovskii, Zh. Éksp. Teor. Fiz. 68, 175 (1975) [Sov. Phys. JETP 41, 85 (1975)]; G. H. Fredrickson and E. Helfand, J. Chem. Phys. 87, 697 (1987).

[28] A similar logarithmic and repulsive singularity is found near a structural transition in nematic liquid crystals: P. Ziherl et al., Phys. Rev. E 61, 5361 (2000).

[29] The mean-field force changes sign as a function of $d$, and this estimate is taken near a negative and local minimum.

[30] In contrast, for a presmectic fluid with monotonic correlation, the Casimir interaction is shown to be of a conventional type: P. Ziherl, Phys. Rev. E 61, 4636 (2000).

[31] D. A. Antelmi et al., J. Phys. II 5, 103 (1995).

(a)

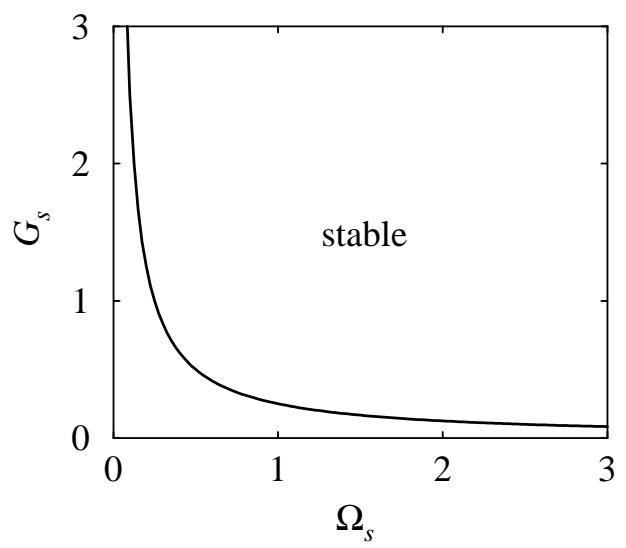

(b)

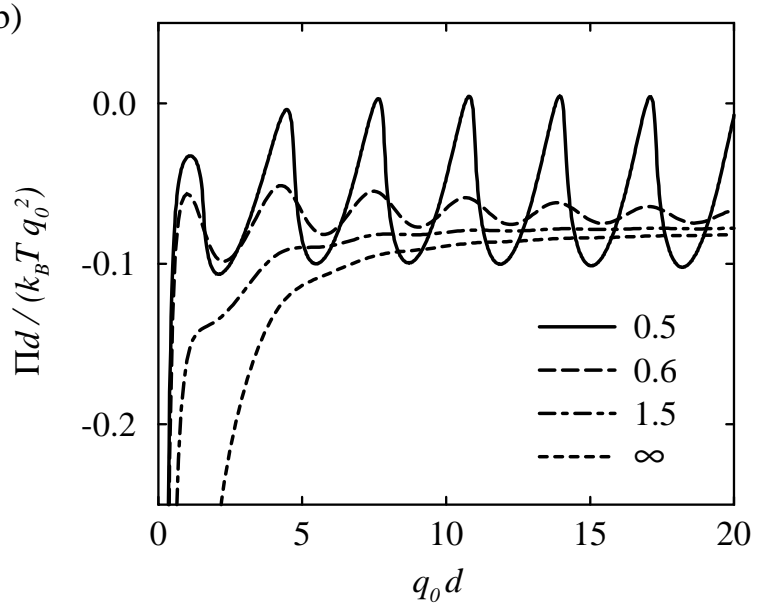

FIG. 2. (a) Region of stability of the mean-field solution $\phi=0$. Outside the region, the surface-induced I-L transition [15] preempts the bulk transition, even if there is only one wall $(d \rightarrow \infty)$. (b) Surface-potential dependence of the Casimir pressure. Plots are for $\left(\Omega_{s}, G_{s}\right)=(0.5,0.5)$, $(0.6,0.6),(1.5,1.5)$, and $(\infty, \infty)$, all at the bulk transition temperature. The point $(0.5,0.5)$ is on the instability line. 